\title{
Resting-state functional connectivity and pitch identification ability in non-musicians
}

\author{
Jiancheng Hou ${ }^{1,2+}$, Chuansheng Chen ${ }^{3 * t}$ and Qi Dong ${ }^{1 *}$ \\ ${ }^{1}$ State Key Lab of Cognitive Neuroscience and Learning, Beijing Normal University, Beijing, China \\ ${ }^{2}$ Department of Psychology, Ohio State University, Columbus, OH, USA \\ ${ }^{3}$ Department of Psychology and Social Behavior, University of California, Irvine, Irvine, CA, USA
}

\section{Edited by:}

Gottfried Schlaug, Beth Israel

Deaconess Medical Center and

Harvard Medical School, USA

\section{Reviewed by:}

Krista Hyde, McGill University, Canada

Vince D. Calhoun, University of New

Mexico, USA

\section{*Correspondence:}

Chuansheng Chen, Department of Psychology and Social Behavior,

University of California, 4566 Social

and Behavioral Sciences Gateway,

Irvine, CA 92697, USA

e-mail: chuansheng.chen@uci.edu;

Qi Dong, State Key Lab of Cognitive

Neuroscience and Learning, Beijing

Normal University, Yingdong

Building, No.19, Xinjiekouwai,

Beijing 100875, China

e-mail: dongqi@bnu.edu.cn

${ }^{\dagger}$ These authors have contributed

equally to this work.
Previous studies have used task-related fMRI to investigate the neural basis of pitch identification (PI), but no study has examined the associations between resting-state functional connectivity (RSFC) and PI ability. Using a large sample of Chinese non-musicians ( $N=320$, with 56 having prior musical training), the current study examined the associations among musical training, PI ability, and RSFC. Results showed that musical training was associated with increased RSFC within the networks for multiple cognitive functions (such as vision, phonology, semantics, auditory encoding, and executive functions). PI ability was associated with RSFC with regions for perceptual and auditory encoding for participants with musical training, and with RSFC with regions for short-term memory, semantics, and phonology for participants without musical training.

Keywords: resting-state functional connectivity, pitch identification, musical training, non-musicians

\section{INTRODUCTION}

Some professional musicians can identify a single musical note quickly and accurately without the benefit of a reference note. They are considered as having absolute pitch (AP) (Levitin, 1999; Parncutt and Levitin, 2001). Neuroimaging studies have shown that AP musicians have a reduced P300 (Nishitani et al., 1998; Hirose et al., 2002), an increased cerebral blood flow (CBF) in the left posterior dorsolateral frontal cortex (Zatorre et al., 1998), and enhanced white matter connectivity and increased fractional anisotropy in the left superior longitudinal fasciculus (Oechslin et al., 2010).

In addition to AP musicians, quasi-AP musicians (i.e., those with a weak form of AP) have also been studied. Using the PET, Wilson et al. $(2006,2009)$ found that, during a pitch identification (PI) test, quasi-AP musicians had significant activations within an extensive right hemisphere network, including the right superior and middle temporal gyri, right dorsolateral prefrontal cortex, right middle and inferior frontal gyri, and right cerebellum. In contrast to the left hemispheric advantage in AP musicians as mentioned in the previous paragraph, Wilson et al. (2009) showed that the right hemisphere was important for PI in quasi-AP musicians.

Thus, far, little is known about the neural basis of PI among non-musicians. Only one study by Schwenzer and Mathiak (2011) collected fMRI data while non-musicians were asked to recognize a single pitch from a set of four frequencies in each trial. The results showed that PI activated the right dorsolateral prefrontal cortex (DLPFC), right medial frontal gyrus, right medial front lobe, bilateral premotor area, and bilateral intraparietal sulcus (IPS). These regions subserve various functions involved in PI: the DLPFC for working memory (Crottaz-Herbette et al., 2004; Grimault et al., 2009); the medial frontal gyrus for error monitoring (Volz et al., 2005); the medial frontal lobe for retaining memory, executive function and attention (Simons and Spiers, 2003; Baird et al., 2006); the premotor area for planning movement (Churchland et al., 2006; Ojakangas et al., 2006); and the IPS for spatial and quantity processing (Castelli et al., 2006; Dormal and Pesenti, 2009; Santens et al., 2010; Schwenzer and Mathiak, 2011). As was the case for quasi-AP musicians, the right hemisphere was important for PI among non-musicians.

Thus, far, no study has examined the role of resting-state functional connectivity (RSFC) among different brain regions in either AP or PI. Resting-state fMRI measures the lowfrequency $(\sim 0.01-0.1 \mathrm{~Hz})$ spontaneous neuronal activity in the brain (Lv et al., 2008) and is believed to reflect neuronal function (Damoiseaux et al., 2006; Fox and Raichle, 2007; Zhang et al., 2014). Thus, far, the only RSFC studies in musicological 
research were those that aimed to characterize the motor systems in musicians. For example, Lv et al. (2008) found significant RSFC between the left and right primary sensorimotor areas in pianists. Luo et al. (2012) found significant RSFC between the motor and multi-sensory cortices (such as visual, auditory, and somatosensory cortices) in musicians, which may reflect their enhanced functional integration among the lower-level perceptual and motor networks as well as the functional consolidation (plasticity) due to long-term music training.

In the current study, we examined the RSFC differences between the non-musicians with and without musical training and the correlations between PI ability and RSFC in a large sample of Chinese non-musicians.

\section{METHODS AND MATERIALS PARTICIPANTS}

Data for the current study came from a larger project conducted with students from Beijing Normal University. 320 undergraduate students (age range: $19-24$ years, mean age $=20.45, S D=1.18$, 191 female and 129 male) had both RSFC data and data on a PI test. Among them, 56 participants (11 males and 45 females) had musical training experience (such as piano, keyboard, violin, accordion, etc.), and 264 participants (118 males and 146 females) had no musical training experience. Table 1 shows participants' basic demographic information.

All participants had normal or corrected-to-normal vision and no history of neurological or psychiatric diseases. They were all right-handed as judged by Snyder and Harris's handedness inventory (Snyder and Harris, 1993). Informed written consent was obtained from all participants before scanning. This study was approved by the Institutional Review Board (IRB) of the State Key Laboratory of Cognitive Neuroscience and Learning at Beijing Normal University.

Table 1 | Characteristics of the participants.

\begin{tabular}{lcc}
\hline Characteristics & Mean (SD) & Range \\
\hline Age (years) & $20.45(1.18)$ & $19-24$ \\
Gender (male/female) & $320(129 / 191)$ & \\
Handedness & All right-handed & \\
Pl accuracy rate (\%) & $37.56(5.13)$ & $4.76-100$ \\
Pl reaction time (millisecond) & $1816.45(423.97)$ & $641-7187$ \\
Music training experience & & \\
Musical training & & \\
Starting age & $10.67(1.44)$ & \\
Gender (male/female) & $56(11 / 45)$ & \\
Pl accuracy rate (\%) & $58.80(5.42)$ & \\
Pl reaction time (millisecond) & $1704.11(321.78)$ & $738.14-4174.42$ \\
No musical training & & $4.76-100$ \\
Gender (male/female) & $264(118 / 146)$ & $641-7187$ \\
Pl accuracy rate (\%) & $31.82(9.49)$ & \\
Pl reaction time (millisecond) & $1859.23(435.64)$ & \\
\hline
\end{tabular}

Note: Standard deviations are shown in parentheses.

\section{PITCH IDENTIFICATION TEST}

The PI test was adapted from the AP test developed by Zatorre (2003). Because the majority of the participants had no formal music training, only seven basic music notes from the fifth octave were used (i.e., C4, D4, E4, F4, G4, A4, and B4, with corresponding frequencies of 261.60, 293.66, 329.63, 349.23, 392.00, 440.00, and $494.88 \mathrm{~Hz}$, respectively). Participants responded by clicking a corresponding key on the computer screen after listening to a note. The seven pitches were randomly presented. Each pitch was presented for $500 \mathrm{~ms}$ twice and was tested three times. The interstimulus interval was $1000 \mathrm{~ms}$. Before the formal test, there was a practice test for $5 \mathrm{~min}$ and participants were given feedback (i.e., they were told which note had been presented), again because most of the participants had no music training. The formal test took about $10 \mathrm{~min}$ to complete and it was conducted without feedback. Accuracy rate and reaction time during the formal test were collected (also see Hou et al., 2014). In current study we analyzed the accuracy rate (see Table $\mathbf{1}$ for mean PI scores, standard deviations, and range; see Figure 1 for distributions of the PI scores by group).

\section{MRI DATA ACQUISITION}

Data were acquired with a $3.0 \mathrm{~T}$ Siemens MRI scanner in the MRI Center of Beijing Normal University. A single-shot T2* weighted gradient echo EPI sequence was used for a brief scan (8 min) which comprised 240 continuous echo planar imaging functional volumes with the following parameters: TR/TE/ $\theta=$ $2000 \mathrm{~ms} / 25 \mathrm{~ms} / 90^{\circ}, \mathrm{FOV}=192 \times 192 \mathrm{~mm}$, matrix $=64 \times 64$, and slice thickness $=3 \mathrm{~mm}$. During the scan, participants laid supine on the scanner bed. Foam pads were used to minimize head motion. Participants were instructed to close their eyes, keep their head still, think about nothing in particular, and just relax. We determined whether participants were awake during scanning by talking to the participants immediately after the session. If they responded immediately and reported that they stayed awake during the scan, we assumed they were awake. Of all the participants in the original larger study, one participant was determined to have slept during scanning, whose data were not included in the database. Anatomical MRI was acquired using a T1-weighted, three-dimensional, gradient-echo pulsesequence (MPRAGE) with $\mathrm{TR} / \mathrm{TE} / \theta=2530 \mathrm{~ms} / 3.09 \mathrm{~ms} / 10^{\circ}$, $\mathrm{FOV}=256 \times 256 \mathrm{~mm}$, matrix $=256 \times 256$, and slice thickness $=1 \mathrm{~mm}$. Two hundred and eight sagittal slices were acquired to provide high-resolution structural images of the whole brain.

\section{REGION OF INTEREST (ROI) SELECTION}

Because the current study did not collect task-related fMRI, we selected seed regions based on a previous task-related fMRI study for PI among non-musicians (Schwenzer and Mathiak, 2011). That study found seven significant ROIs (with MNI coordinates indicated): right dorsolateral prefrontal cortex $(x=40, y=$ $20, z=36)$, right medial frontal gyrus $(x=4, y=18, z=50)$, right medial frontal lobe $(x=34, y=48, z=8)$, left premotor area $(x=-30, y=-6, z=50)$, right premotor area $(x=24$, $y=-2, z=52)$, left intraparietal sulcus $(x=-36, y=-34$, $z=40)$, and right intraparietal sulcus $(x=40, y=-40, z=44)$. The radius was $6 \mathrm{~mm}$. Please see all ROIs in Figure 2. 

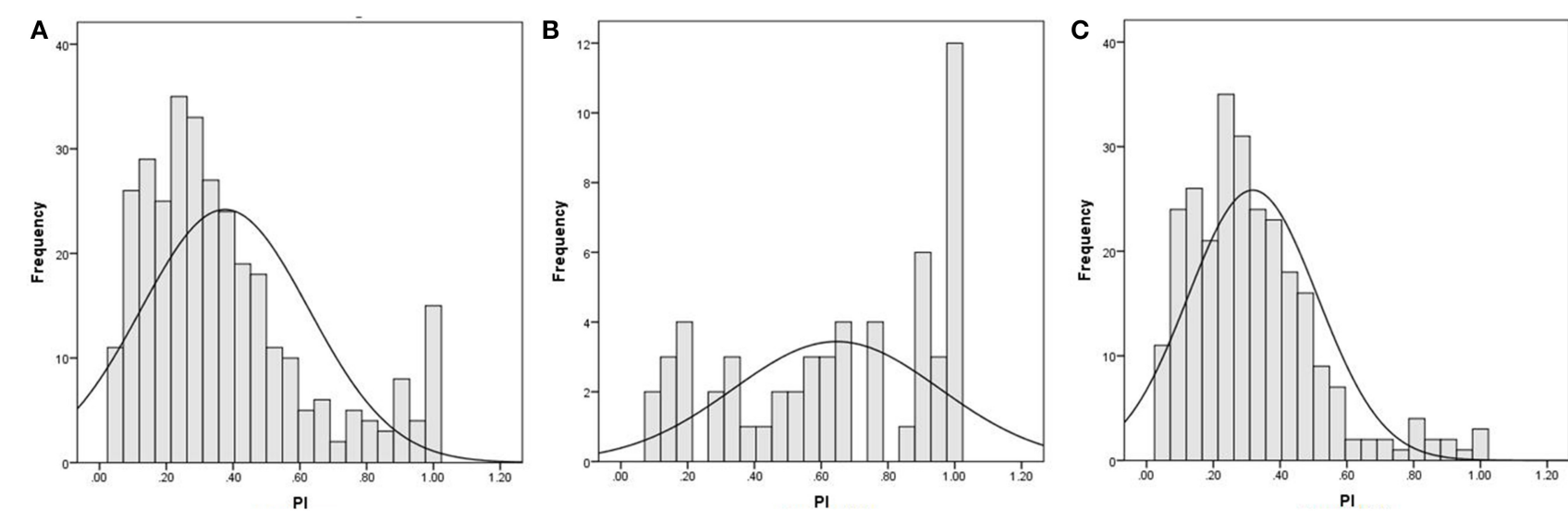

FIGURE 1 | The distribution of participants' accuracy rate on the pitch identification test. (A) the total sample of 320 participants; (B) the participants with musical training; (C) the participants without musical training.

\section{DATA PREPROCESSING}

Image preprocessing was carried out using Data Processing Assistant for Resting-State fMRI (DPARSF) (http://www.nitrc. org/projects/dparsf/) version 2.2. DPARSF is a convenient plugin software based on Statistical Parametric Mapping (SPM) and Resting-State fMRI Data Analysis Toolkit (REST) (http:// www.restfmri.net). The Digital Imaging and Communications in Medicine (DICOM) files were first arranged and the parameters (such as time points, TR, slice number, voxel size et al.) were then set. DPARSF then produced the preprocessed data (with slice timing, realignment, normalization, and smoothing) and the results of functional connectivity (FC), regional homogeneity (ReHo), amplitude of low-frequency fluctuation (ALFF) and fractional ALFF (fALFF). The current study used the FC analysis. The first 10 volumes were discarded to allow the magnetisation to approach a dynamic equilibrium, and for the participants to get used to the scanner noise. No participants showed head motion above $3.0 \mathrm{~mm}$ of maximal translation (in any direction of $x, y$, or $z$ ) and $2.5^{\circ}$ of maximal rotation throughout the course of scanning (Yan et al., 2009). Data pre-processing, including slice timing, realignment, normalization, smoothing, regressing out head motion parameters (using a least squares approach and a 6-parameter spatial transformation), and spatial normalization to the Montreal Neurological Institute (MNI) template (resampling voxel size of $3 \times 3 \times 3 \mathrm{~mm}$ ), were conducted using SPM8 and DPARSF version 2.2 (Yan and Zang, 2010; Kuhn et al., 2012). A spatial filter of $5 \mathrm{~mm}$ FWHM (full-width at half maximum) was used.

\section{STATISTICAL ANALYSIS}

SPSS 16.0 version was used to analyze the behavioral data. For the RSFC analysis, the Resting-State fMRI Data Analysis Toolkit (REST) (http://www.restfmri.net) was used (Yan and Zang, 2010; Song et al., 2011). To examine the music training effects, we conducted independent-sample $t$-tests on a whole brain $Z$-value map between the participants with and without musical training. Within each group, we then correlated the $Z$-value map with the PI score. Gender was included as a covariate. Monte
Carlo simulations were performed using the AFNI AlphaSim program for multiple comparison correction. By iterating the process of random image generation, spatial correlation of voxels, thresholding, and cluster identification, the program provides an estimate of the overall significance level achieved for various combinations of individual voxel probability threshold and cluster size threshold (Bennett et al., 2009; Wu et al., 2011). Using this program, a threshold correction adjustment was used with a voxel-wise $p<0.05,1000$ simulations, cluster size $>212$ $\left(5724 \mathrm{~mm}^{3}\right.$ ). Because the interpretations of negative RSFC (or anti-correlations) are still being debated and their neuronal basis is unclear (e.g., Weissenbacher et al., 2009; but for recent developments, see Chai et al., 2012; Liang et al., 2012), we focused on our analyses on positive RSFC. In addition, we focused on positive behavioral correlates of these RSFC and presented the negative behavioral correlates in the Supplemental Online Materials.

\section{RESULTS}

The mean accuracy rate on the PI test was $37.56 \%(S D=5.13)$, ranging from 4.76 to $100 \%$ (15 participants had 100\%) (see Figure 1A). For the participants with musical training, the mean accuracy rate on the PI test was $65.19 \%(S D=3.09)$, ranging from 9.52 to $100 \%$ (12 participants had 100\%, Figure 1B). For the participants without musical training, the mean was $31.82 \%$ $(S D=9.49)$, ranging from 4.76 to $100 \%$ (3 participants had $100 \%$, Figure 1C). The group difference was significant, $t_{(318)}=$ $10.21, p<0.001$.

We then compared group differences in RSFC between the seed regions and other brain areas. Compared to participants without musical training, those with musical training showed stronger RSFC between the right dorsolateral prefrontal cortex seed and the bilateral superior temporal gyri and right inferior parietal lobule; between the right medial frontal gyrus seed and the right precuneus; between the left premotor area seed and the right cerebellum, right superior medial frontal gyrus, and left pars triangularis; between 


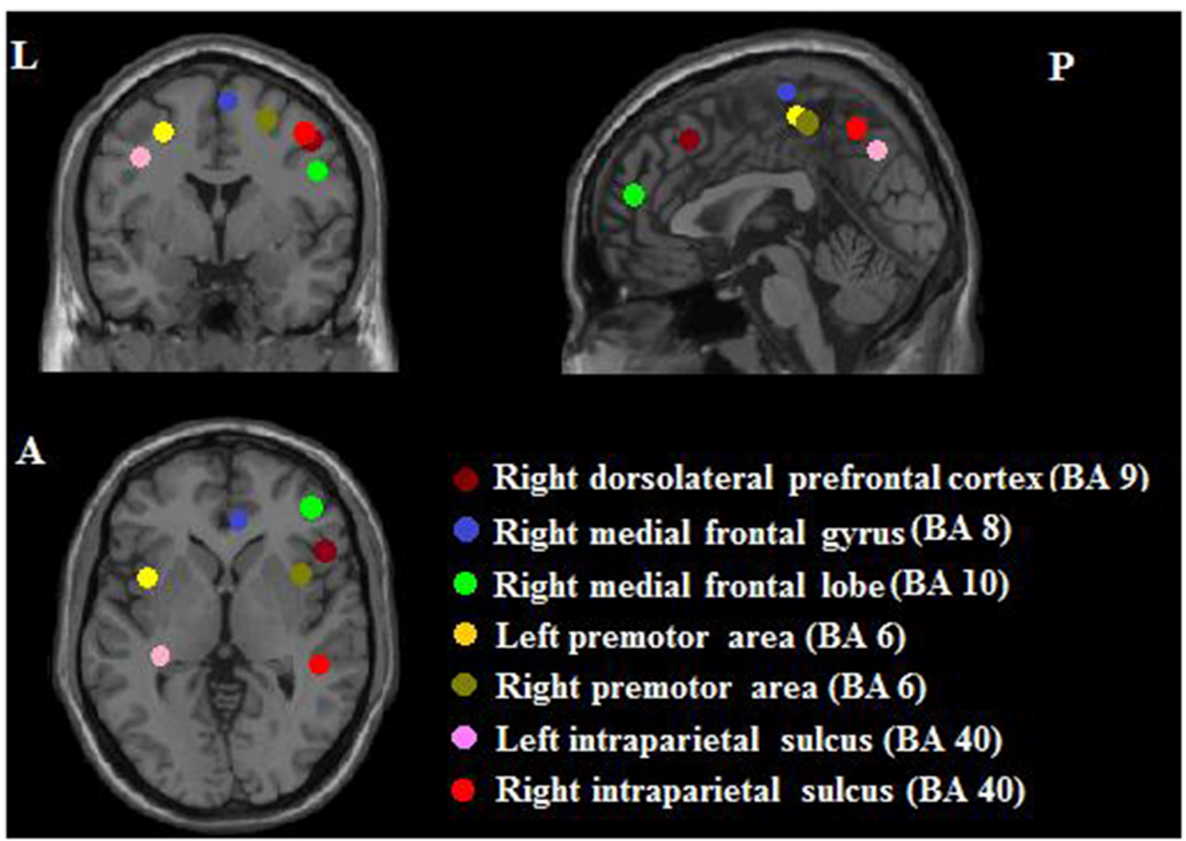

FIGURE 2 | Seven seed ROIs. L, left; A, anterior; P, posterior.

Table 2 | Positive differences in RSFC between participants with and without musical training.

\begin{tabular}{|c|c|c|c|c|c|c|c|}
\hline \multirow[t]{2}{*}{ ROI seeds } & \multirow[t]{2}{*}{ Cluster location } & \multirow[t]{2}{*}{ BA } & \multicolumn{3}{|c|}{ Peak (MNI) } & \multirow[t]{2}{*}{ Cluster size } & \multirow[t]{2}{*}{$\boldsymbol{t}$} \\
\hline & & & $\boldsymbol{x}$ & $y$ & $z$ & & \\
\hline \multirow[t]{3}{*}{ Right dorsolateral prefrontal cortex } & Right superior temporal gyrus & 48 & 42 & -27 & 66 & 252 & 4.15 \\
\hline & Right inferior parietal lobule & 40 & 54 & -45 & 39 & 257 & 3.23 \\
\hline & Left superior temporal gyrus & 48 & -18 & 14 & -6 & 246 & 3.35 \\
\hline Right medial frontal gyrus & Right precuneus & 5 & 0 & -48 & 63 & 457 & 4.39 \\
\hline \multirow[t]{3}{*}{ Left premotor area } & Right cerebellum & & 6 & -60 & -45 & 224 & 4.49 \\
\hline & Right superior medial frontal gyrus & 8 & 9 & 30 & 60 & 400 & 3.73 \\
\hline & Left pars triangularis & 45 & -33 & 42 & -6 & 213 & 4.07 \\
\hline \multirow[t]{5}{*}{ Right premotor area } & Right inferior temporal gyrus & 20 & 63 & -18 & -21 & 616 & 4.40 \\
\hline & Right pars triangularis & 45 & 45 & 45 & -15 & 320 & 4.33 \\
\hline & Right cerebellum & & 42 & -66 & -45 & 241 & 4.09 \\
\hline & Left inferior temporal gyrus & 20 & -60 & -18 & -24 & 2005 & 4.79 \\
\hline & Left middle frontal gyrus & 9 & -39 & 18 & 48 & 1429 & 5.25 \\
\hline Left intraparietal sulcus & Left middle frontal gyrus & 46 & -33 & 18 & 45 & 380 & 4.29 \\
\hline
\end{tabular}

Note: AlphaSim corrected $p<0.05$, cluster size $>212$.

the right premotor area seed and the bilateral inferior temporal gyrus, right pars triangularis, right cerebellum, and left middle frontal gyrus; and between the left intraparietal sulcus seed and the left middle frontal gyrus. Detailed information of these results are shown in Table 2 and Figure 3.

Correlations between RSFC and PI were obtained for each group of participants. For participants with musical training, positive correlates of PI ability included RSFC between the bilateral premotor area seeds and the left cerebellum (see Table 3 and
Figure 4). For participants without musical training, positive correlates of PI ability included RSFC between the right dorsolateral prefrontal cortex seed and the right cerebellum, and between the left premotor area seed and the bilateral inferior parietal lobule, right pars triangularis, and left superior temporal gyrus (see Table 3 and Figure 5).

In addition to the positive relationships between RSFC and cognitive performance, our analysis also revealed several significant negative relationships between RSFC and musical training and between RSFC and PI ability. These results are 


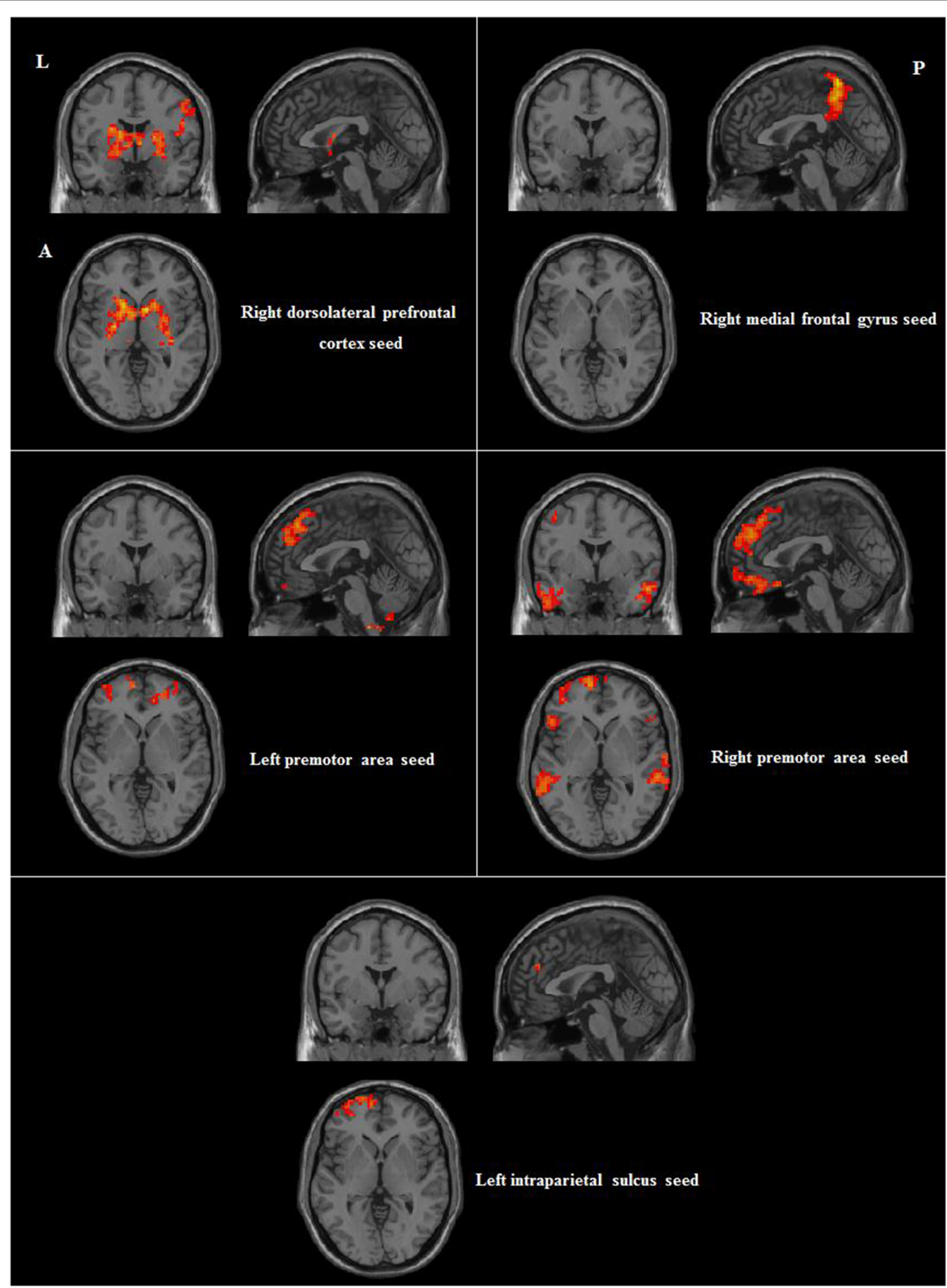

FIGURE 3 | Differences in positive RSFC between participants with and without musical training (AlphaSim corrected $p<0.05$, cluster size $>212$ ). L, left; A, anterior; P, posterior.

presented in Supplementary Tables S1, S2 and Supplementary Figures S1-S3. Finally, because our ROIs were selected based on a previous task fMRI study, which might have missed important seed regions, we conducted a whole-brain analysis across the Automated Anatomical Labeling (AAL) ROIs. Additional associations between RSFC and musical training and between RSFC and PI ability were identified. These results and a brief discussion are presented in Supplementary Tables S3, S4.

\section{DISCUSSION}

Using a large sample of non-musicians, the current study aimed to examine how RSFC was associated with musical training and PI ability.

\section{MUSIC TRAINING AND RSFC}

Whole-brain analysis revealed significant RSFC differences between participants with and without musical training. First, 
Table 3 | Positive correlations between RSFC and PI for the two groups.

\begin{tabular}{|c|c|c|c|c|c|c|c|}
\hline ROI seeds & Cluster location & BA & \multicolumn{3}{|c|}{ Peak (MNI) } & Cluster size & $r$ \\
\hline \multicolumn{8}{|c|}{ PARTICIPANTS WITH MUSICAL TRAINING } \\
\hline Left premotor area & Left cerebellum & & -33 & -87 & -27 & 725 & 0.58 \\
\hline Right premotor area & Left cerebellum & & -30 & -87 & -27 & 317 & 0.46 \\
\hline \multicolumn{8}{|c|}{ PARTICIPANTS WITHOUT MUSICAL TRAINING } \\
\hline & Right inferior parietal lobule & 40 & 33 & -48 & 42 & 251 & 0.22 \\
\hline & Left superior temporal gyrus & 48 & -48 & 21 & 27 & 227 & 0.23 \\
\hline & Left inferior parietal lobule & 7 & -30 & -66 & 54 & 323 & 0.28 \\
\hline
\end{tabular}

Note: AlphaSim corrected $p<0.05$, cluster size $>212$.

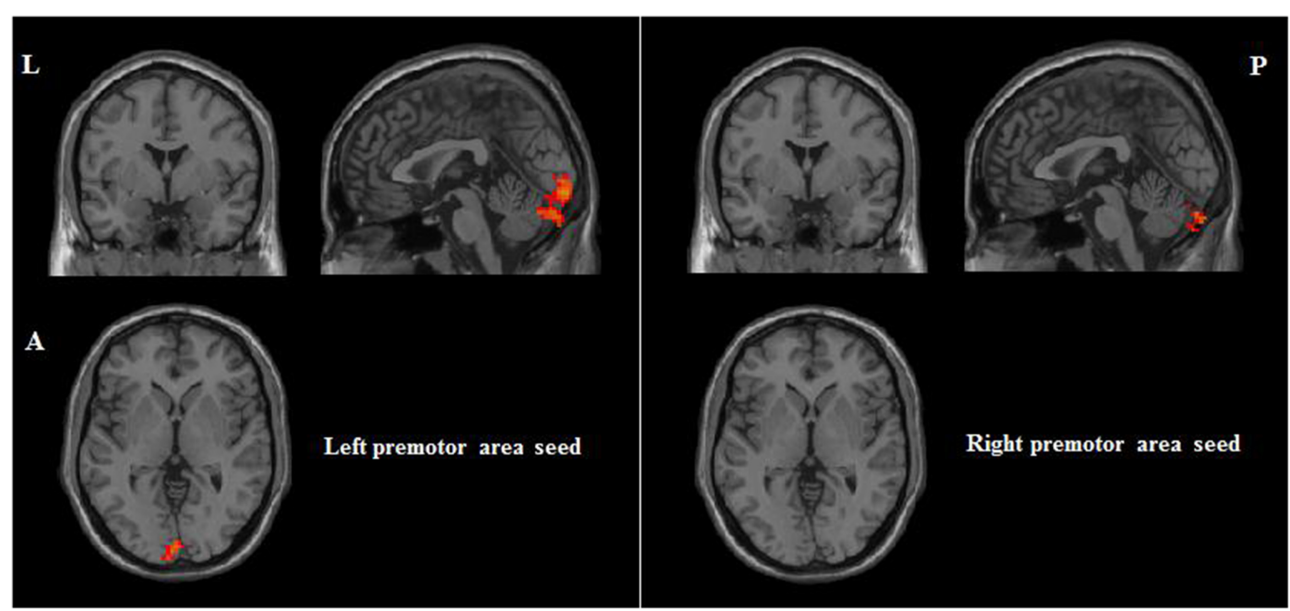

FIGURE 4 | Positive correlations between RSFC and PI for participants with musical training (AlphaSim corrected $p<0.05$, cluster size $>212$ ). L, left; A, anterior; P, posterior.

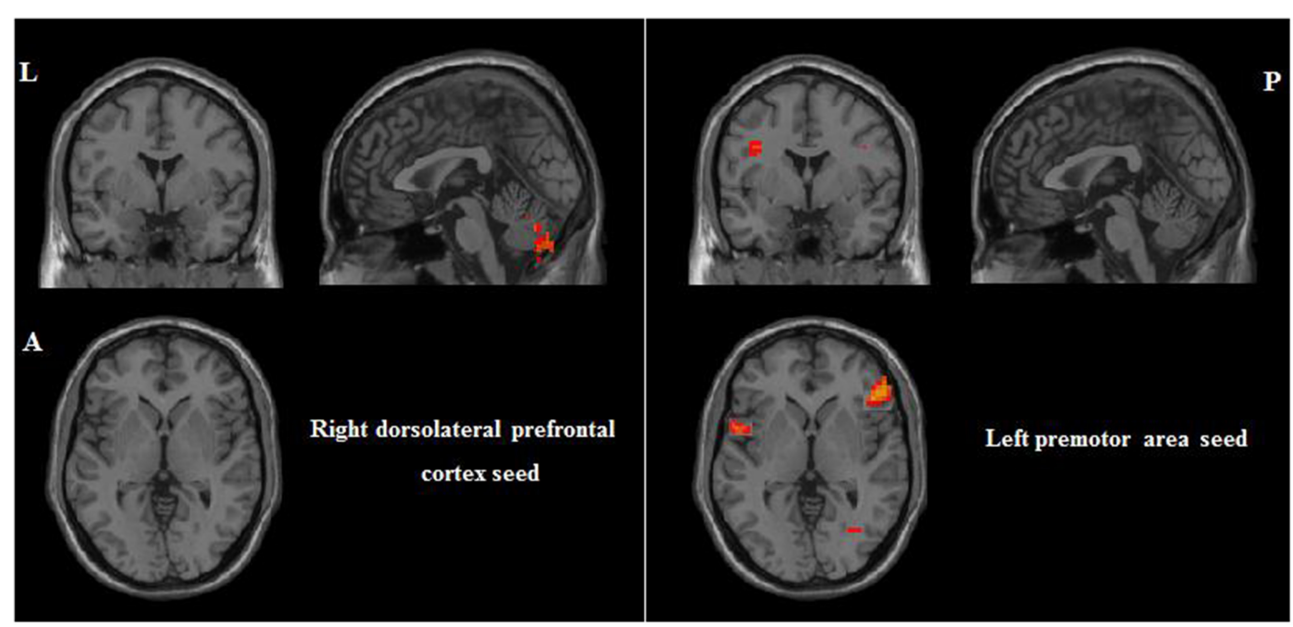

FIGURE 5 | Positive correlations between RSFC and PI for participants without musical training group (AlphaSim corrected $p<0.05$, cluster size $>$ 212). $L$, left; $A$, anterior; $P$, posterior. 
musical training was associated with increased functional connectivity linking the right DLPFC seed to the bilateral superior temporal gyrus and right inferior parietal lobule (the latter is within the default mode network). These results are aligned with previous findings of music training effects on these regions' functions in language processing. For example, musical training has been found to improve phonological and semantic processing in the superior temporal gyrus (Platel et al., 2003; Lai et al., 2014), phonological processing in the inferior parietal lobule (Bermudez and Zatorre, 2005; Bermudez et al., 2009; Lai et al., 2014), and executive functions in the inferior parietal lobule (Bermudez and Zatorre, 2005).

Second, musical training was associated with increased functional connectivity linking the right medial frontal gyrus seed to the right precuneus. The precuneus, another region within the default mode network, is responsible for musical memory, imagery and emotion (Demorest et al., 2010). Previous research has shown that musical training can improve this region's visual "mental imagery" in a pitch change task (Platel et al., 1999; Meister et al., 2004).

Third, the participants with musical training showed significantly increased functional connectivity linking the bilateral premotor area seeds to the regions that subserve the functions of object recognition (bilateral inferior temporal gyrus, Bogousslavsky et al., 1987; Heywood et al., 1995; Olson et al., 2007), auditory processing and speech (bilateral inferior temporal gyrus, bilateral pars triangularis, McGuire et al., 1995; Onitsuka et al., 2004; Kaplan et al., 2010; Romanski, 2012), the maintenance and selective retrieval of memory components (left middle frontal gyrus, Bermudez and Zatorre, 2005), executive functions (right superior medial frontal cortex, Talati and Hirsch, 2005), and behavior or movement control and auditory encoding (right cerebellum, Gaab et al., 2003; Petacchi et al., 2005; Schulze et al., 2009). This cluster of strengthened RSFC may reflect the benefits of music training on cognitive abilities such as vision, kinesthesia, motion perception, verbal processing, and even higher cognitive functions (Zatorre and Beckett, 1989; Zhou, 2004; Bermudez and Zatorre, 2005).

Finally, the participants with musical training showed significantly increased functional connectivity linking the left intraparietal sulcus seed to the left middle frontal gyrus. The IPS is mainly responsible for musical, spatial, and quantity processing (Cappelletti et al., 2007; Husain and Nachev, 2007; Offen et al., 2010; Cheng et al., 2013). Foster and Zatorre (2010) found an increased activation in the intraparietal sulcus for music note processing, perhaps involving the visual-spatial mapping scheme (i.e., imagining notes on a staff or using a spatial coding for their relative pitch height) during pitch processing (also see Zhou, 2004; Rusconi et al., 2005; Williamson et al., 2011). Schulze et al. (2009) found that the middle frontal gyrus was involved in the tonal working memory. It seems that music training strengthened connectivities between the above two regions and consequently pitch perception and memory (Zatorre and Beckett, 1989; Gaab et al., 2003; Zhou, 2004).

\section{RSFC AND PI ABILITY}

For participants without musical training, there were two main findings. First, there was a positive correlation between the right dorsolateral prefrontal cortex seed and the right cerebellum. The cerebellum, traditionally viewed as a motor structure, is found to be active in a wide variety of sensory and cognitive tasks. Schulze et al. (2009) found that a music pitch memory task elicited cerebellar activations in both AP and non-AP musicians (also see Gaab et al., 2003). It has also been found to be involved in higher cognitive processes such as working memory (Baddeley, 2003; Marvel et al., 2012) and multimodal encoding (Stewart et al., 2003; Cullen, 2012; Billings et al., 2014). In a meta-analysis of 15 PET and fMRI auditory studies, Petacchi et al. (2005) found that a variety of auditory tasks consistently activated the cerebellum. The RSFC-PI relationship between the dorsolateral prefrontal cortex and the cerebellum perhaps reflects the role of this functional connectivity in pitch memory and discrimination (Gaab et al., 2003).

Second, there were significant correlations between PI ability and RSFC linking the left premotor area seed to the right pars triangularis, left superior temporal gyrus, and bilateral inferior parietal lobule. These three regions are responsible for language processing: the pars triangularis and superior temporal gyrus for semantic processing (Kaplan et al., 2010; Romanski, 2012; Lai et al., 2014), and the superior temporal gyrus and inferior parietal lobule for phonological processing (Hickok and Poeppel, 2004, 2007; Scott and Wise, 2004; Bermudez and Zatorre, 2005; Limb et al., 2006; Bermudez et al., 2009; Romanski, 2012). As mentioned earlier, these regions have been shown to be affected by music training. Interestingly, the RSFC between these regions and the DLPFC seed was associated with music training as reported in the previous section, but the RSFC between these regions and the premotor area seed was associated with PI ability for participants without music training. Further research is needed to explicate these differential associations.

For the participants with musical training, whose sample size was relatively small, there were only two significant associations: between the bilateral premotor area seeds and the left cerebellum. These functional connectivities suggest that better PI ability may rely on stronger connection between auditory encoding at the cerebellum to movement preparation and control at the premotor area (e.g., Gaab et al., 2003; Petacchi et al., 2005; Schulze et al., 2009).

\section{LIMITATIONS OF THE CURRENT STUDY}

Several limitations of the current study need to be noted. First, in order to accommodate the non-musician participants, we used a PI test that had a restricted range of notes and included practice trials. Thus, our results cannot be generalized to AP musicians. Second, because we did not include task-related fMRI for PI in this study, we relied on a previous study for ROI selection (as well as AAL for additional ROIs in the supplementary result), which might have missed important seed regions for our participants. Third, our sample size of the participants with music training was small and thus had less statistical power, which might have contributed to the divergent results between the two groups of participants. Fourth, our data were correlational, so it was not clear whether there were causal relations among musical training, PI ability, and RSFC. Fifth, because there was no effective way to monitor whether participants slept during the resting-state scanning other than immediate post-scan self-report, it was uncertain 
how many participants might have drifted away from wakefulness toward sleep, a concern that has been raised recently about RSFC studies (Tagliazucchi and Laufs, 2014). Finally, our participants were Chinese whose native language is a tonal language. Previous research has shown that speakers of tonal languages might have an advantage in AP or PI ability (Deutsch et al., 2004; Gandour et al., 1998; also see Bidelman et al., 2013, for an advantage of Cantonese speakers). Therefore, our results need to be replicated among speakers of non-tonal languages.

\section{SUMMARY}

With a large sample of Chinese non-musicians, the current study compared RSFC differences between participants with and without musical training and correlated RSFC with PI ability within each group. The results showed that musical training was associated with increased RSFC within the networks for multiple cognitive functions, such as vision, phonology, semantics, auditory encoding, and executive functions. Moreover, PI ability was associated with RSFC with regions for perceptual and auditory encoding for participants with musical training, and with RSFC with regions for short-term memory, semantics, and phonology for participants without musical training.

\section{ACKNOWLEDGMENTS}

This study was supported by the 111 Project from the Ministry of Education of China (B07008). We thank all graduate research assistants who helped us with data collection.

\section{SUPPLEMENTARY MATERIAL}

The Supplementary Material for this article can be found online at: http://www.frontiersin.org/Journal/10.3389/fnins. 2015.00007/abstract

\section{REFERENCES}

Baddeley, A. D. (2003). Working memory: looking back and looking forward. Nat. Rev. Neurosci. 4, 829-839. doi: 10.1038/nrn1201

Baird, A., Dewar, B., Critchley, H., Gilbert, S. J., Dolan, R. J., and Cipolotti, L. (2006). Cognitive functioning after medial frontal lobe damage including the anterior cingulate cortex: a preliminary investigation. Brain Cognit. 60, 166-175. doi: 10.1016/j.bandc.2005.11.003

Bennett, C. M., Wolford, G. L., and Miller, M. B. (2009). The principled control of false positives in neuroimaging. Soc. Cogn. Affect. Neurosci. 4, 417-422. doi: 10.1093/scan/nsp053

Bermudez, P., Lerch, J. P., Evans, A. C., and Zatorre, R. J. (2009). Neuroanatomical correlates of musicianship as revealed by cortical thickness and voxel-based morphometry. Cereb. Cortex 19, 1583-1596. doi: 10.1093/cercor/bhn196

Bermudez, P., and Zatorre, R. J. (2005). Conditional associative memory for musical stimuli in nonmusicians: implications for absolute pitch. J. Neurosci. 25, 7718-7723. doi: 10.1523/JNEUROSCI.1560-05.2005

Bidelman, G. M., Hutka, S., and Moreno, S. (2013). Tone language speakers and musicians share enhanced perceptual and cognitive abilities for musical pitch: evidence for bidirectionality between the domains of language and music. PLoS ONE 8:e60676. doi: 10.1371/journal.pone.0060676

Billings, G., Piasini, E., Lorincz, A., Nusser, Z., and Silver, R. A. (2014). Network structure within the cerebellar input layer enables lossless sparse encoding. Neuron 83, 960-974. doi: 10.1016/j.neuron.2014.07.020

Bogousslavsky, J., Miklossy, J., Deruaz, J. P., Assal, A., and Regli, F. (1987). Lingual and fusiform gyri in visual processing: a clinico-pathologic study of superior altitudinal hemianopia. J. Neurol. Neurosurg. Psychiatry 50, 607-614. doi: 10.1136/jnnp.50.5.607

Cappelletti, M., Barth, H., Fregni, F., Spelke, E. S., and Pascual-Leone, A. (2007). rTMS over the intraparietal sulcus disrupts numerosity processing. Exp. Brain Res. 179, 631-642. doi: 10.1007/s00221-006-0820-0
Castelli, F., Glaser, D. E., and Butterworth, B. (2006). Discrete and analoque quantity processing in the parietal lobe: a functional MRI study. Proc. Natl. Acad. Sci. U.S.A. 103, 4693-4698. doi: 10.1073/pnas.0600444103

Chai, X. J., Castanon, A. N., Ongur, D., and Whitfield-Gabrieli, S. (2012). Anticorrelations in resting state networks without global signal regression. Neuroimage 59, 1420-1428. doi: 10.1016/j.neuroimage.2011.08.048

Cheng, G. L., Tang, J., Walsh, V., Butterworth, B., and Cappelletti, M. (2013). Differential effects of left parietal theta-burst stimulation on order and quantity processing. Brain Stimul. 6, 160-165. doi: 10.1016/j.brs.2012.04.005

Churchland, M. M., Yu, B. M., Ryu, S. I., Santhanam, G., and Shenoy, K. V. (2006) Neural variability in premotor cortex provides a signature of motor preparation. J. Neurosci. 26, 3697-3712. doi: 10.1523/JNEUROSCI.3762-05.2006

Crottaz-Herbette, S., Anagnoson, R. T., and Menon, V. (2004). Modality effects in verbal working memory: differential prefrontal and parietal responses to auditory and visual stimuli. Neuroimage 21, 340-351. doi: 10.1016/j.neuroimage.2003.09.019

Cullen, K. E. (2012). The vestibular system: multimodal integration and encoding of self-motion for motor control. Trends Neurosci. 35, 185-196. doi: 10.1016/j.tins.2011.12.001

Damoiseaux, J. S., Rombouts, S. A., Barkhof, F., Scheltens, P., Stam, C. J., Smith, S. M., et al. (2006). Consistent resting-state networks across healthy subjects. Proc. Natl. Acad. Sci. U.S.A. 103, 13848-12853. doi: 10.1073/pnas.0601417103

Demorest, S. M., Morrison, S. J., Stambaugh, L. A., Beken, M., Richards, T. L., and Johnson, C. (2010). An fMRI investigation of the cultural specificity of music memory. Soc. Cogn. Affect. Neurosci. 5, 282-291. doi: 10.1093/scan/nsp048

Deutsch, D., Henthorn, T., and Dolson, M. (2004). Absolute pitch, speech, and tone language: some experiments and a proposed framework. Music Percept. 21, 339-356. doi: 10.1525/mp.2004.21.3.339

Dormal, V., and Pesenti, M. (2009). Common and specific contributions of the intraparietal sulci to numerosity and length processing. Hum. Brain Mapp. 30, 2466-2476. doi: 10.1002/hbm.20677

Foster, N. E., and Zatorre, R. J. (2010). A role for the intraparietal sulcus in transforming musical pitch information. Cereb. Cortex 20, 1350-1359. doi: 10.1093/cercor/bhp199

Fox, M. D., and Raichle, M. E. (2007). Spontaneous fluctuations in brain activity observed with functional magnetic resonance imaging. Nat. Rev. Neurosci. 8, 700-711. doi: 10.1038/nrn2201

Gaab, N., Gaser, C., Zaehle, T., Jancke, L., and Schlaug, G. (2003). Functional anatomy of pitch memory-an fMRI study with sparse temporal sampling. Neuroimage 19, 1417-1426. doi: 10.1016/S1053-8119(03)00224-6

Gandour, J., Wong, D., and Hutchins, G. (1998). Pitch processing in the human brain is influenced by language experience. Neuroreport 9, 2115-2119. doi: 10.1097/00001756-199806220-00038

Grimault, S., Robitaille, N., Grova, C., Lina, J. M., Dubarry, A. S., and Jolicoeur, P. (2009). Oscillatory activity in parietal and dorsolateral prefrontal cortex during retention in visual short-term memory: additive effects of spatial attention and memory load. Hum. Brain Mapp. 30, 3378-3392. doi: 10.1002/hbm.20759

Heywood, C. A., Gaffan, D., and Cowey, A. (1995). Cerebral achromatopsia in monkeys. Eur. J. Neurosci. 7, 1064-1073. doi: 10.1111/j.14609568.1995.tb01093.x

Hickok, G., and Poeppel, D. (2004). Dorsal and ventral streams: a framework for understanding aspects of the functional anatomy of language. Cognition 92, 67-99. doi: 10.1016/j.cognition.2003.10.011

Hickok, G., and Poeppel, D. (2007). The cortical organization of speech processing. Nat. Rev. Neurosci. 8, 393-402. doi: 10.1038/nrn2113

Hirose, H., Kubota, M., Kimura, I., Ohsawa, M., Yumoto, M., and Sakakihara, Y. (2002). People with absolute pitch process tones with producing P300. Neurosci. Lett. 330, 247-250. doi: 10.1016/S0304-3940(02)00812-1

Hou, J. C., Chen, C. S., Wang, Y. P., Liu, Y. Y., He, Q. H., Li, J., et al. (2014) Superior pitch identification ability is associated with better executive functions. Psychomusicol. Music Mind Brain 24, 136-146. doi: 10.1037/a0036963

Husain, M., and Nachev, P. (2007). Space and the parietal cortex. Trends Cogn. Sci. 11, 30-36. doi: 10.1016/j.tics.2006.10.011

Kaplan, E., Naeser, M. A., Martin, P. I., Ho, M., Wang, Y., Baker, E., et al. (2010). Horizontal portion of arcuate fasciculus fibers track to pars opercularis, not pars triangularis, in right and left hemispheres: a DTI study. Neuroimage 52, 436-444. doi: 10.1016/j.neuroimage.2010.04.247

Kuhn, S., Vanderhasselt, M. A., De Raedt, R., and Gallinat, J. (2012). Why ruminators won't stop: the structural and resting state correlates of 
rumination and its relation to depression. J. Affect. Disord. 141, 352-360. doi: 10.1016/j.jad.2012.03.024

Lai, M. C., Lombardo, M. V., Ecker, C., Chakrabarti, B., Suckling, J., Bullmore, E. T., et al. (2014). Neuroanatomy of individual differences in language in adult males with autism. Cereb. Cortex. doi: 10.1093/cercor/bhu211. [Epub ahead of print].

Levitin, D. J. (1999). Absolute pitch: self-reference and human memory. Int. J. Comput. Anticipat. Syst. 4, 255-266.

Liang, Z., King, J., and Zhang, N. (2012). Anticorrelated resting-state functional connectivity in awake rat brain. Neuroimage 59, 1190-1199. doi: 10.1016/j.neuroimage.2011.08.009

Limb, C. J., Kemeny, S., Ortigoza, E. B., Rouhani, S., and Braun, A. R. (2006). Left hemispheric lateralization of brain activity during passive rhythm perception in musicians. Anat. Rec. A Discov. Mol. Cell. Evol. Biol. 288, 382-389. doi: 10.1002/ar.a.20298

Luo, C., Guo, Z. W., Lai, Y. X., Liao,W., Liu, Q., Kendrick, K. M., et al. (2012). Musical training induces functional plasticity in perceptual and motor networks: insights from resting-state fMRI. PLoS ONE 7:e36568. doi: 10.1371/journal.pone.0036568

Lv, Y. T., Yang, H., Wang, D., Li, S., Han, Y., Zhu, C. Z., et al. (2008). Correlations in spontaneous activity and gray matter density between left and right sensoritmotor areas of pianists. Neuroreport 19, 631-634. doi: 10.1097/WNR.0b013e3282fa6da0

Marvel, C. L., Faulkner, M. L., Strain, E. C., Mintzer, M. Z., and Desmond, J. E. (2012). An fMRI investigation of cerebellar function during verbal working memory in methadone maintenance patients. Cerebellum 11, 300-310. doi: 10.1007/s12311-011-0311-0

McGuire, P. K., Silbersweig, D. A., Wright, I., Murray, R. M., David, A. S., Frackowiak, R. S., et al. (1995). Abnormal monitoring of inner speech: a physiological basis for auditory hallucinations. Lancet 346, 596-600. doi: 10.1016/S0140-6736(95)91435-8

Meister, I. G., Krings, T., Foltys, H., Boroojerdi, B., Muller, M., Topper, R., et al. (2004). Playing piano in the mind-an fMRI study on music imagery and performance in pianists. Brain Res. Cogn. Brain Res. 19, 219-228. doi 10.1016/j.cogbrainres.2003.12.005

Nishitani, N., Nagamine, T., Fujiwara, N., Yazawa, S., and Shibasaki, H. (1998). Cortical-hippocampal auditory processing identified by magnetoencephalography. J. Cogn. Neurosci. 10, 231-247. doi: 10.1162/089892998562672

Oechslin, M. S., Imfeld, A., Loenneker, T., Meyer, M., and Jancke, L. (2010). The plasticity of the superior longitudinal fasciculus as a function of musical expertise: a diffusion tensor imaging study. Front. Hum. Neurosci. 3:76. doi: 10.3389/neuro.09.076.2009

Offen, S., Gardner, J. L., Schluppeck, D., and Heeger, D. J. (2010). Differential roles for frontal eye fields (FEFs) and intraparietal sulcus (IPS) in visual working memory and visual attention. J. Vis. 10:28. doi: 10.1167/10.11.28

Ojakangas, C. L., Shaikhouni, A.,Friehs, G. M., Caplan, A. H., Serruya, M. D., Saleh, M., et al. (2006). Decoding movement intent from human premotor cortex neurons for neural prosthetic applications. J. Clin. Neurophysiol. 23, 577-584. doi: 10.1097/01.wnp.0000233323.87127.14

Olson, I. R., Plotzker, A., and Ezzyat, Y. (2007). The Enigmatic temporal pole: a review of findings on social and emotional processing. Brain $130(\mathrm{Pt} 7)$, 1718-1731. doi: 10.1093/brain/awm052

Onitsuka, T., Shenton, M. E., Salisbury, D. F., Dickey, C. C., Kasai, K., Toner, S. K., et al. (2004). Middle and inferior temporal gyrus gray matter volume abnormalities in chronic schizophrenia: an MRI study. Am. J. Psychiatry 161, 1603-1611. doi: 10.1176/appi.ajp.163.12.2103

Parncutt, R., and Levitin, D. J. (2001). “Absolute pitch,” in New Grove Dictionary of Music and Musicians, ed S. Sadie (London, UK: MacMillan), 37-39.

Petacchi, A., Laird, A. R., Fox, P. T., and Bower, J. M. (2005). Cerebellum and auditory function: an ALE meta-analysis of functional neuroimaging studies. Hum. Brain Mapp. 25, 118-128. doi: 10.1002/hbm.20137

Platel, H., Baron, J. C., Desgranges, B., Bernard, F., and Eustache, F. (2003). Semantic and episodic memory of music are subserved by distinct neural networks. Neuroimage 20, 244-256. doi: 10.1016/S1053-8119(03)00287-8

Platel, H., Price, C., Baron, J. C., Wise, R., Lambert, J., Frackowiak, R. S., et al. (1999). The structural components of music perception. A functional anatomical study. Brain 120, 229-243. doi: 10.1093/brain/120.2.229

Romanski, L. M. (2012). Integration of faces and vocalizations in ventral prefrontal cortex: implications for the evolution of audiovisual speech. Proc. Natl. Acad. Sci. U.S.A. 109(Suppl. 1), 10717-10724. doi: 10.1073/pnas.1204335109
Rusconi, E., Kwan, B., Giordano, B., Umilta, C., and Butterworth, B. (2005). The mental space of pitch height. Ann. N.Y. Acad. Sci. 1060, 195-197. doi: 10.1196/annals. 1360.056

Santens, S., Roggeman, C., Fias, W., and Verguts, T. (2010). Number processing pathways in human parietal cortex. Cereb. Cortex 20, 77-88. doi: 10.1093/cercor/bhp080

Schulze, K., Gaab, N., and Schlaug, G. (2009). Perceiving pitch absolutely: comparing absolute and relative pitch possessors in a pitch memory task. BMC Neurosci. 10:106. doi: 10.1186/1471-2202-10-106

Schwenzer, M., and Mathiak, K. (2011). Numeric aspects in pitch identification: an fMRI study. BMC Neurosci. 12:26. doi: 10.1186/1471-2202-12-26

Scott, S. K., and Wise, R. J. (2004). The functional neuroanatomy of prelexical processing in speech perception. Cognition 92, 13-45 doi: 10.1016/j.cognition.2002.12.002

Simons, J. S., and Spiers, H. J. (2003). Prefrontal and medial temporal lobe interactions in long-term memory. Nat. Rev. Neurosci. 4, 637-648. doi: $10.1038 /$ nrn 1178

Snyder, P. J., and Harris, L. J. (1993). Handedness, sex, and familial sinistrality effects on spatial tasks. Cortex 29, 115-134. doi: 10.1016/S0010-9452(13) 80216-X

Song, X. W., Dong, Z. Y., Long, X. Y., Li, S., Zuo, X. N., Zhu, C. Z., et al. (2011). REST: a toolkit for resting-state functional magnetic resonance imaging data processing. PLoS ONE 6:e25031. doi: 10.1371/journal.pone. 0025031

Stewart, L., Henson, R., Kampe, K., Walsh, V., Turner, R., and Frith, U. (2003). Brain changes after learning to read and play music. Neuroimage 20, 71-83. doi: 10.1016/S1053-8119(03)00248-9

Tagliazucchi, E., and Laufs, H. (2014). Decoding wakefulness levels from typical fMRI resting state data reveals reliable drifts between wakefulness and sleep. Neuron 82, 695-708. doi: 10.1016/j.neuron.2014. 03.020

Talati, A., and Hirsch, J. (2005). Functional specialization within the medial frontal gyrus for perceptual go/no-go decisions based on "what," "when," and "where" related information: an fMRI study. J. Cogn. Neurosci. 17, 981-993. doi: $10.1162 / 0898929054475226$

Volz, K. G., Schubotz, R. I., and von Cramon, D. Y. (2005). Variants of uncertainty in decision-making and their neural correlates. Brain Res. Bull. 67, 403-412. doi: 10.1016/j.brainresbull.2005.06.011

Weissenbacher, A., Kasess, C., Gerstl, F., Lanzenberger, R., Moser, E., and Windischberger, C. (2009). Correlations and anticorrelations in restingstate functional connectivity MRI: a quantitative comparison of preprocessing strategies. Neuroimage 47, 1408-1416. doi: 10.1016/j.neuroimage.2009. 05.005

Williamson, V. J., Cocchini, G., and Stewart, L. (2011). The relationship between pitch and space in congenital amusia. Brain Cognit. 76, 70-76. doi: 10.1016/j.bandc.2011.02.016

Wilson, S., Lusher, D., Wan, C., Dudgeon, P., and Reutens, D. (2006). "Imaging the neurocognitive components of pitch naming: insights from quasi-absolute pitch," in Proceedings of 9th International Conference on Music Perception and Cognition (ICMPC 2006) (Liege, Belgium), 825-833.

Wilson, S. J., Lusher, D., Wan, C. Y., Dudgeon, P., and Reutens, D. C. (2009). The neurocognitive components of pitch processing: insights from absolute pitch Cereb. Cortex 19, 724-732. doi: 10.1093/cercor/bhn121

Wu, J. T., Wu, H. Z., Yan, C. G., Chen, W. X., Zhang, H. Y., He, Y., et al. (2011). Aging-related changes in the default mode network and its anticorrelated networks: a resting-state fMRI study. Neurosci. Lett. 504, 62-67. doi: 10.1016/j.neulet.2011.08.059

Yan, C. G., Liu, D. Q., He, Y., Zou, Q. H., Zhu, C. Z., Zuo, X. N., et al. (2009). Spontaneous brain activity in the default mode network is sensitive to different resting-state conditions with limited cognitive load. PLoS ONE 4:e5743. doi: 10.1371/journal.pone.0005743

Yan, C. G., and Zang, Y. F. (2010). DPARSF: a Matlab toolbox for "pipeline" data analysis of resting-state fMRI. Front. Syst. Neurosci. 4:13. doi: $10.3389 /$ fnsys. 2010.00013

Zatorre, R. J. (2003). Absolute pitch: a model for understanding the influence of genes and development on neural and cognitive function. Nat. Neurosci. 6, 692-695. doi: 10.1038/nn1085

Zatorre, R., and Beckett, C. (1989). Multiple coding strategies in the retention of musical tones by possessors of absolute pitch. Mem. Cognit. 17, 582-589. doi: 10.3758/BF03197081 
Zatorre, R. J., Perry, D. W., Beckett, C. A., Westbury, C. F., and Evans, A. C. (1998). Functional anatomy of musical processing in listeners with absolute pitch and relative pitch. Proc. Natl. Acad. Sci. U.S.A. 95, 3172-3177. doi: 10.1073/pnas.95.6.3172

Zhang, M. X., Li, J., Chen, C. S., Xue, G., Lu, Z. L., Mei, L. L., et al. (2014). Restingstate functional connectivity and reading abilities in first and second languages. Neuroimage 84, 546-553. doi: 10.1016/j.neuroimage.2013.09.006

Zhou, H. H. (2004). The Music and the Words of Related Expressions (in Chinese). Beijing: College of Center Music Press.

Conflict of Interest Statement: The authors declare that the research was conducted in the absence of any commercial or financial relationships that could be construed as a potential conflict of interest.
Received: 13 June 2014; accepted: 08 January 2015; published online: 11 February 2015.

Citation: Hou J, Chen C and Dong Q (2015) Resting-state functional connectivity and pitch identification ability in non-musicians. Front. Neurosci. 9:7. doi: 10.3389/fnins. 2015.00007

This article was submitted to Auditory Cognitive Neuroscience, a section of the journal Frontiers in Neuroscience.

Copyright (C) 2015 Hou, Chen and Dong. This is an open-access article distributed under the terms of the Creative Commons Attribution License (CC BY). The use, distribution or reproduction in other forums is permitted, provided the original author(s) or licensor are credited and that the original publication in this journal is cited, in accordance with accepted academic practice. No use, distribution or reproduction is permitted which does not comply with these terms. 